\title{
PROBLEMA EN EL NUMERO DE PARCHES DE CIRROS NUCALES EN PECES DEL GENERO CALLICLINUS GILL 1860 (OSTEICHTHYES, LABRISOMIDAE)
}

\author{
PROBLEMS IN THE AMOUNT OF CEPHALIC CIRRI PATCHES IN FISHES \\ OF GENUS CALLICLINUS GILL 1860 (OSTEICHTHYES, \\ LABRISOMIDAE)
}

\author{
Germán Pequeño R. \& Sylvia Sáez B. \\ Instituto de Zoología “Ernst F. Kilian”, Universidad Austral de Chile, Casilla 567, Valdivia, Chile. \\ Email: gpequeno@uach.cl
}

RESUMEN

La existencia de peces del género Calliclinus con menos de tres parches de cirros nucales genera problemas sistemáticos y taxonómicos, especialmente confusión con el género Auchenionchus Gill, uno de cuyos caracteres diagnósticos indica dos parches. Se analiza el problema con 543 ejemplares y se concluye que pese a este problema, los taxa descritos son válidos.

Palabras claves: Labrisomidae, Calliclinus, cirros nucales, taxonomía, Chile.

\section{ABSTRACT}

The existence of fishes of the genus Calliclinus with less than three patches of nape cirri, produce systematic and taxonomic problems, mainly confussion with the genus Auchenionchus Gill, in which one of the diagnostic characteristics is to have two patches. The problem is analyzed on the basis of 543 specimens. The main conclusion is that both described taxa are valid, in spite of this problem.

Keywords: Labrisomidae, Calliclinus, nape cirri, taxonomy, Chile.

\section{INTRODUCCION}

El género Calliclinus Gill 1860 agrupa a dos especies: C. geniguttatus (Valenciennes 1836) y $C$. nudiventris Cervigón \& Pequeño 1979 (Cervigón et al. 1979, Inzunza \& Pequeño 1988) ambas de la familia Labrisomidae (Pequeño 1989). Son especies simpátricas, que habitan generalmente pozas intermareales y se distribuyen desde Antofagasta hasta Punta Arenas y desde Caleta Leandro hasta Chiloé, respectivamente (Cervigón et al. 1979, Inzunza \& Pequeño 1988).
Se caracterizan por poseer escamas en la región ventral y postaxilar, en el caso de genigutttatus, y carecer de éstas en nudiventris, además de algunos caracteres merísticos como: el número de espinas en la aleta dorsal, radios en la aleta anal, aletas pélvicas, número de escamas en la línea lateral y presencia de tres parches de cirros nucales (Cervigón et al. 1979, Hubbs 1952, Stephens \& Springer 1974), caracteres que permiten la diferenciación de otros labrisómidos. Desde la descripción original de C. nudiventris y análisis de muestras de $C$. geniguttatus, se sabe de ejemplares anómalos o con malformaciones 
(Cervigón et al. 1979), como lo son uno o dos parches de cirros nucales, lo cual contradice la diagnosis genérica (Hubbs 1952, Stephens \& Springer 1974), provocando incertidumbre en su taxonomía, pese a que los caracteres específicos permanecen bien definidos. La presencia de dos parches de cirros nucales corresponde también a uno de los caracteres diagnósticos de otro género de labrisómido: Auchenionchus Gill 1860 (Stephens \& Springer, op cit.).

Con el objetivo de dilucidar este problema taxonómico e interpretar el significado de la variabilidad del número de parches de cirros nucales presentes en genigutattus y nudiventris, el presente trabajo tiene por finalidad:

1. Revisar los caracteres merísticos y lepidológicos (ver Material y Métodos) seleccionados de Calliclinus, efectuando una comparación entre los ejemplares con anormalidades (con uno o dos parches de cirros nucales) y sin ellas y, 2. Determinar si existe alguna relación entre la aparición del carácter anómalo y la distribución geográfica de geniguttatus y nudiventris.

El análisis de los caracteres diagnósticos de Calliclinus, contribuirá a esclarecer la variabilidad que pudiera presentar este carácter tan especial, sugiriendo una explicación tentativa, que permitirá a la vez corregir cualquier deficiencia existente en las claves taxonómicas, ayudando al reconocimiento específico y, por ende, evitar futuros errores.

\section{MATERIAL Y METODOS}

Se examinó una muestra total de 543 especímenes de Calliclinus de la colección de peces marinos del Instituto de Zoología de la Universidad Austral de Chile (IZUA-PM), de los cuales 46 ejemplares correspondían a $C$. nudiventris provenientes desde Pto. Montt ( $\left.41^{\circ} 28^{\prime} \mathrm{S}\right)$ hasta Yaldad $\left(43^{\circ} 08^{\prime} \mathrm{S}\right)$ y 497 ejemplares de $C$. geniguttatus cuya distribución estuvo comprendida entre Los Molles (IV Región) y Yaldad.

El estudio se basó en una primera etapa sobre todos los ejemplares de ambas especies, con la finalidad de separar aquellos que presentaran uno o dos parches de cirros nucales. Una vez seleccionados los ejemplares, se procedió a la etapa de análisis merístico. Los recuentos se efectuaron en el lado izquierdo de cada pez, fueron: número de espinas y radios de las aletas dorsales, pectoral, pélvica, anal y caudal. Para el recuento de los poros cefálicos se contó desde el extremo anterior de la cabeza hacia atrás, en sentido cefalocaudal del ejemplar, además se consideró análogamente el criterio utilizado por Smith-Vaniz \& Springer (1971). Se contó también el número de escamas de la línea lateral, sobre y bajo ésta. Tales análisis merísticos se compararon con cinco ejemplares de cada especie elegidos al azar, que no presentaran la anormalidad en los cirros nucales. A continuación se indica las tallas y el material estudiado en cada especie, longitud total (LT), longitud estándar (LE), número de catálogo y número del o los ejemplares en la muestra respectiva: a) C. geniguttatus (58-119 mm LT; $46-132 \mathrm{~mm}$ de LE): IZUA PM 23 (1 y 2), 227 (17), 293 (105, 106 y 107), 1421 (1); b) C. nudiventris (47-85 mm LT ; 38-72 mm LE): IZUA PM 993 (2 y 3), 1395 (6 y 50), 1407 (2).

Se efectuó un estudio morfológico de los canales cefálicos de la línea lateral, indicando además el número de los poros cefálicos, los cuales fueron cotejados con la misma muestra utilizada en el estudio merístico. Para facilitar tal análisis, se examinaron ejemplares diafanizados de ambas especies (IZUA PM 173 y 293). Se realizó también un estudio comparativo lepidológico (morfológico y merístico) entre los ejemplares anómalos y normales. Para ello se obtuvieron escamas de tres áreas del lado izquierdo del cuerpo: área alfa ubicada bajo la primera aleta dorsal y sobre la línea lateral; área beta, entre la punta de la aleta pectoral y la línea lateral; área gama, localizada en la línea lateral en la zona inmediatamente anterior al pedúnculo caudal (Fig. 1A). Luego, se procedió a depositarlas entre dos portaobjetos, debidamente rotuladas, para luego ser observadas bajo lupa con un aumento de $40 \mathrm{X}$.

\section{RESULTADOS}

\section{REVISION DE CARACTERES EN CALLICLINUS}

Este estudio se efectuó en cuatro aspectos:

1.1 Selección de ejemplares anómalos

a) C. geniguttatus. De un total de 497 especímenes, sólo siete ejemplares $(\mathrm{LE}=45-220 \mathrm{~mm}$ ) presentaron dos parches de cirros nucales, lo que corresponde a un $1.4 \%$ de la muestra analizada. 
b) $C$. nudiventris. De un total de 46 ejemplares sólo tres especímenes $(\mathrm{LE}=45-87 \mathrm{~mm})$ presentaron el carácter anómalo, correspondiendo a un $6.5 \%$ de la muestra. En ninguna de las muestras analizadas hubo ejemplares sin cirros nucales, situación que hasta ahora sólo ha sido conocida por Cervigón et al. (1979).

\subsection{Análisis merísticos de ejemplares anómalos y normales}

a) C. geniguttatus. Los rangos obtenidos en el análisis merístico comparativo no evidenciaron diferencias importantes entre los ejemplares anómalos y aquellos con tres parches de cirros nucales. Los rangos de estos análisis en los individuos anómalos fueron: aleta dorsal XXII-XXV, 11-12; 14-15 en la aleta pectoral; 3 en la pélvica; II, 19-20 en la anal y 13 en la aleta caudal. En los ejemplares normales los rangos registrados fueron: aleta dorsal XXIII-XXIV, 12-13; 15 en la aleta pectoral; 3-4 en la pélvica; II, 17-20 en la anal y 13 en la aleta caudal.

b) C. nudiventris. Tampoco hubo diferencias importantes en los rangos obtenidos entre los especímenes anómalos y normales. En los individuos anómalos los rangos merísticos fueron: aleta dorsal XXII-XXIII, 11-12; 14-15 en la aleta pectoral; 3 en la pélvica; II, 17-19 en la anal y 13 en la aleta caudal. En los ejemplares normales, los rangos registrados fueron: aleta dorsal XXII-XXIII, 11-12; 15 en la aleta pectoral; 2-3 en la pélvica; II, 17-18 en la anal y 13 en la aleta caudal.

\subsection{Canales y poros cefálicos de ejemplares anómalos y normales}

En ambas especies se observó fácilmente, tanto en los ejemplares anómalos como normales, la ramificación de los canales cefálicos de la línea lateral con gran cantidad de poros. La primera ramificación comienza en la porción anterior del hueso nasal dando origen al canal supraorbital (Figs. 1B y 1B, b). Cada canal supraorbital continúa dorsolateralmente hacia atrás bordeando el ojo, curvándose hacia abajo y dividiéndose en una rama inferior formando el canal infraorbital (Fig. 1B y 1C, c), y en una rama posterior, el canal pterótico (Fig. $1 \mathrm{~B}, \mathrm{a})$, el cual se dirige hacia atrás al canal de la línea lateral. A su vez, este último se proyecta primero hacia el preopérculo formando el canal preopercular
(Fig. 1C, d) y, dorsomedialmente hacia la región supratemporal dando origen al canal supratemporal (Fig. 1C, a). Finalmente, el canal preopercular se curva anteroventralmente juntándose con el canal mandibular y formando el canal preopérculo-mandibular (Fig. 1B, d).

La cuenta de poros supratemporales en ejemplares anómalos de $C$. nudiventris, varió entre 6-7; 6 en la región pterótica y supraorbital; $10-11$ poros en el canal infraorbital; 14 en el preopercular y seis poros mandibulares, además de la presencia de dos poros adicionales situados por debajo del primer poro mandibular (debajo de la sínfisis) (Figs. 1D y 1E). En los ejemplares normales de nudiventris la cuenta de los poros fue: supratemporales 6; 5-6 en la región pterótica; 6 poros supraorbitales; 12 poros en el canal infraorbital; 12-14 poros preoperculares y seis poros mandibulares. Como en los ejemplares anómalos, también fue posible reconocer dos poros adicionales situados por debajo del primer poro mandibular (Figs. 1D y 1E). En geniguttatus, la cuenta de los poros varió entre: 5-7 en la región supraorbital; $10-11$ poros en el canal infraorbital; 4 en el canal pterótico; 10-14 en el preopercular y 5-6 poros mandibulares, observándose además los dos poros adicionales debajo de la sínfisis registrados en la especie anterior; y 5-6 poros en la región supratemporal. En los individuos normales fue: 6-7; 5-6 en la región pterótica y supraorbital; 8-11 poros en el canal infraorbital; 12-14 en el preopercular y seis poros mandibulares (Figs. 1D y 1E).

\subsection{Análisis lepidológico}

a) Morfología lepidológica. Se encontraron diferencias lepidológicas muy leves entre las tres distintas escamas (alfa, beta, gama) extraídas a los ejemplares anómalos y los ejemplares normales. En el ejemplar anómalo de mayor talla $(\mathrm{LT}=255 \mathrm{~mm})$ se observó una escama alfa comparativamente más ancha que en los ejemplares normales.

En todos los ejemplares analizados se observaron escamas pseudocicloídeas.

i) Comparación intraespecífica en nudiventris. Para esta comparación se escogieron los ejemplares PM 1395 (LT = 55 mm) y PM 993 (2) (LT = 56 mm). Hubo desigualdades muy sutiles entre los tres tipos de escamas, principalmente en los bordes de la parte anterior (insertada) de los ejemplares anómalos, donde las escamas eran de bordes más redondeados 

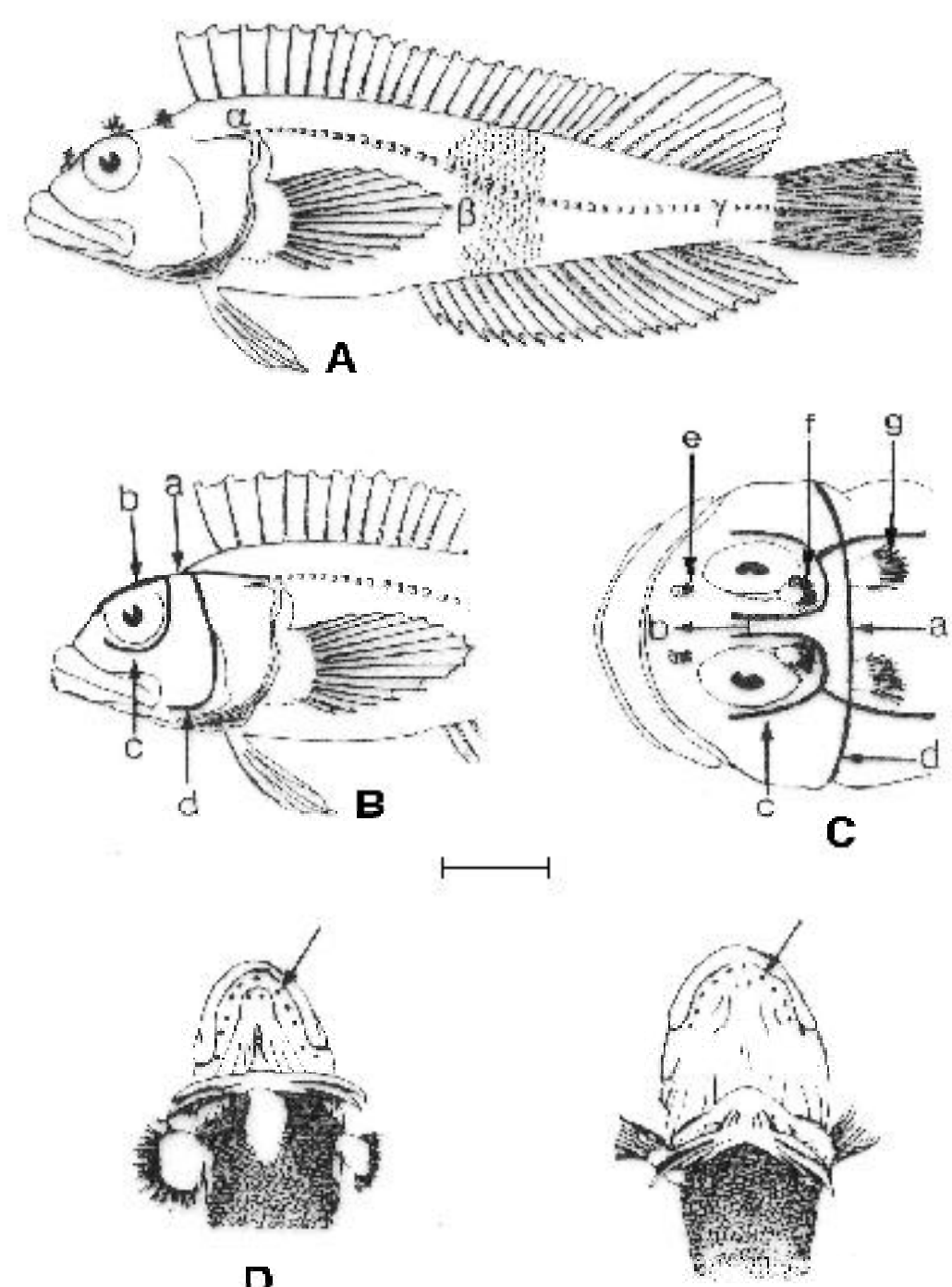

$\mathbf{D}$

FIgURa 1 A-E. La escala indica $1 \mathrm{~cm}$ en todos los casos: A. Areas de extracción de las escamas en Calliclinus: a = escamas alfa, $b=$ escamas beta, g= escama gama (Modificado de Cervigón et al. 1979). B. Vista lateral de los canales cefálicos observados en Calliclinus: a) Pterótico. b) Supraorbital. c) Infraorbital. d) Preoperculomandibular. C. Vista dorsal de los canales cefálicos y cirros observados en Calliclinus: Canales a) Supratemporal. b) Supraorbital. c) Infraorbital. d) Preopercular; Cirros e) Nasal. f) Supraorbitales y g) Nucales (Modificado de Cervigón et al. 1979). D. Vista ventral de la distribución de los poros mandibulares de $C$. nudiventris (Modificado de Inzunza \& Pequeño 1988). E. Vista ventral de la distribución de los poros mandibulares de C. geniguttatus (Modificado de Inzunza \& Pequeño 1988). La flecha señala los poros mandibulares.

Figure 1 A-E. Scale indicates $1 \mathrm{~cm}$ in all cases: A. Sampling areas for scales in Calliclinus: $\mathrm{a}=\mathrm{alfa}$ scales, $\mathrm{b}=$ beta scales, $\mathrm{g}=$ gama scales (Modified from Cervigón et al. 1979). B. Lateral view of cephalic channels seen in Calliclinus: a) Pterotic b) Supraorbital. c) Infraorbital. d) Preoperculomandibular. C. Dorsal view of cephalic cephalic channels and cirri as seen in Calliclinus: Channels a) Pterotic b) Supraorbital. c) Infraorbital. d) Preoperculomandibular; Cirri e) Nasal. f) Supraorbital and g) Nape (Modified from Cervigón et al. 1979). D. Ventral view of mandibular pores distribution in C. nudiventris (Modified from Inzunza \& Pequeño 1988). E. Ventral view of mandibular pores arrangement in C. geniguttatus (Modified from Inzunza \& Pequeño 1988). The arrow indicates the mandibular pores. 
mientras que los normales se caracterizaban por presentar bordes más rectos.

ii) Comparación intraespecífica engeniguttatus. Para esta comparación se escogieron los ejemplares PM 1421 (LT=55 mm) y PM 227 (7) (LT=58 mm). Las observaciones efectuadas esta especie, presentaron la misma tendencia que nudiventris.

iii) Comparación interespecífica. Los pares de ejemplares elegidos fueron: PM 1405 ( $C$. geniguttatus anormal LT=89 mm) con PM 1407 (C. nudiventris normal LT=85 $\mathrm{mm}$ ). En el caso de los ejemplares anómalos de nudiventris se compararon con: PM 293 (C. geniguttatus normal $\mathrm{LT}=119 \mathrm{~mm}$ ) con PM 266 (C.nudiventris anómalo $\mathrm{LT}=105 \mathrm{~mm}$ ).
Sólo en las escamas gama de ambas especies se pudo apreciar diferencias respecto a lo observado en la comparación interespecífica. Mientras que los ejemplares anómalos de geniguttatus presentaban bordes más rectos respecto a los especímenes normales, en los individuos anómalos de nudiventris dicha escama se caracterizó por tener bordes más redondeados que los ejemplares normales de geniguttatus.

b) Merística. Se registraron ciertas desigualdades al comparar los rangos del recuento de escamas por arriba y en la línea lateral de los ejemplares de nudiventris. En geniguttatus las desigualdades se detectaron en el recuento de escamas situadas abajo y en la línea lateral (Tabla I).

TABLA I. Rangos obtenidos en el recuento de escamas sobre, bajo y en la línea lateral registradas en los ejemplares con dos parches (anómalos) y tres parches (normales) de C. nudiventris y C. geniguttatus.

TABLE I. Range values obtained in scales counting over, below and on the lateral line, recorded in the specimens with two patches of cirri (anomalous) and three patches (normal) of $C$. nudiventris and C. geniguttatus.

\begin{tabular}{lcccc}
\hline \multicolumn{4}{c}{ ANOMALOS } & \multicolumn{2}{c}{ NORMALES } \\
\hline $\mathrm{N}^{\mathrm{o}}$ escamas & $\begin{array}{c}\text { C. nudiventris } \\
(\mathrm{LS}=45-87 \mathrm{~mm})\end{array}$ & $\begin{array}{c}\text { C. geniguttatus } \\
\mathrm{LS}=45-220 \mathrm{~mm})\end{array}$ & $\begin{array}{c}\text { C. } \text { nudiventris } \\
(\mathrm{LS}=38-72 \mathrm{~mm})\end{array}$ & $\begin{array}{c}\text { C. } \text { geniguttatus } \\
(\mathrm{LS}=46-132 \mathrm{~mm})\end{array}$ \\
\hline $\begin{array}{l}\text { Sobre la } \\
\text { linea lateral }\end{array}$ & $5-7$ & $6-12$ & $7-8$ & 8 \\
$\begin{array}{l}\text { Bajo la } \\
\text { línea lateral }\end{array}$ & $11-13$ & $11-22$ & $11-13$ & $13-14$ \\
En la línea lateral & $43-45$ & $43-53$ & $42-43$ & $43-44$ \\
\hline
\end{tabular}

II. NUMERO DE PARCHES DE CIRROS NUCALES VS. DISTRIBUCIONGEOGRAFICA.

El carácter anormal en ambas especies se registró principalmente en Calbuco (Pto. Montt), donde también se observó un número comparativamente mayor de ejemplares con tres parches de cirros nucales respecto a las otras localidades de captura.

\section{DISCUSION}

El registro de los especímenes con dos parches de cirros nucales tanto en las muestras analizadas en el presente trabajo como en aquellos analizados previamente por Cervigón et al. (1979), es una característica considerada como anormal, ya que se opone a lo descrito en la literatura (Hubbs 1952, Stephens \& Springer 1974). Los antecedentes obtenidos por Cervigón et al. (op. cit.) no pueden ser comparados con el presente estudio, ya que esos autores no separaron los ejemplares anómalos de aquellos que presentaban tres parches de cirros.

Respecto al análisis merístico, se destaca la dificultad que existió en todos los especímenes analizados para ver la espina en la aleta pélvica, que en el caso de geniguttatus es descrita por Stephens \& Springer (1974). Esto se resolvió con el examen de ejemplares 
diafanizados, donde se pudo constatar que dicha espina se encuentra muy pegada a los radios (confundiéndola con uno de ellos), además de ser relativamente pequeña, lo que provoca que en un examen táctil no sea posible detectarla fácilmente.

En cuanto a los poros y canales cefálicos, pese a ser un carácter taxonómico utilizado en peces y en algunas familias del Suborden Blennioidei (Stephens \& Springer 1974, Castillo \& Pequeño 1998), en Calliclinus no existe información con la cual se pueda comparar lo caracterizado en geniguttatus y nudiventris. Se destaca que no hubo diferencias en forma o cantidad de poros, entre los especímenes anómalos y normales.

La importancia de un estudio lepidológico entre los ejemplares de Calliclinus se basa fundamentalmente sobre el análisis de las escamas de diversos grupos de peces que ha ayudado a dilucidar problemas sistemáticos y taxonómicos, ya que se presentan variaciones entre familias, géneros y especies (Batts 1964, Hughes 1981, Miles \& Gaglione 1992, Roberts 1993). En nuestros análisis comparativos, se observó que tanto en los datos merísticos como en los morfológicos, pese a encontrar ciertas desigualdades, éstas fueron menores. La diferencia morfológica más evidente fue encontrada en un ejemplar anómalo de geniguttatus $(\mathrm{LT}=255 \mathrm{~mm})$ en el ancho de la escama alfa. Esto se explica ya que el crecimiento de la escama está relacionado con la longitud del ejemplar (Batts 1964), de ahí lo relevante de utilizar ejemplares con talla similar para efectuar una comparación intra o interespecífica. En los rangos registrados en la cuenta de las escamas en la línea lateral de los ejemplares anómalos de geniguttatus, el valor máximo (correspondiente al ejemplar de mayor talla) es notoriamente superior respecto a lo observado en los ejemplares normales, situación que por el momento no tiene justificación. Respecto a la distribución geográfica, se destaca la presencia conjunta de ejemplares con el carácter normal con aquellos que presentan dos parches de cirros, sólo en una localidad (Pto. Montt), mientras que el resto de los especímenes anómalos se registraron en Mehuín y en Chiloé, no quedando claro o descartando, por el momento, una relación entre la presencia conjunta de ejemplares anómalos o normales con su distribución geográfica. Para dilucidar esta problemática es necesario obtener un número mayor de ejemplares de ambas especies, ampliando las localidades de captura, tarea para posibles estudios futuros.
La variabilidad en el número de parches registrado en Calliclinus y que se manifiesta por un número menor de parches (dos parches), pese a que se registra en un bajo porcentaje, debe ser considerada con cautela al momento de emplear las claves taxonómicas que comúnmente ayudan al reconocimiento genérico. Esta variación no significa la presencia de un nuevo taxón, ya que los caracteres de las especies permanecen sin variación, confirmando lo señalado por Cervigón et al. (1979). Pero, podría estar revelando un parentesco estrecho con Auchenionchus, si se supone que la disminución del número de parches constituye un carácter derivado. Así, Auchenionchus debería ser considerado una forma más nueva que Calliclinus. Si por el contrario, el aumento del número de parches es considerado una derivación, Calliclinus pasaría a representar una forma más moderna.

La función o rol de los cirros nucales es poco conocida o simplemente ignorada, aunque hay antecedentes en especies de la familia Blenniidae donde está claro que cirros como los supraorbitales cumplen una función sensorial y forman parte de un complejo mecanismo sensorial-conductual (von Bartheld \& Meyer 1985, Kotrschal 1999). Sin embargo, no es posible compararlos con las estructuras aquí analizadas pues se trata de otro tipo de cirros.

Es probable que sólo una alteración genética o malformación, quizás a una temprana etapa del desarrollo del individuo, explique la variabilidad de los cirros nucales. Será necesario considerar estudios genéticos (por ej. secuencia de ADN), como aquellos ya realizados en peces del mismo Suborden (Stepien 1992, Stepien et al. 1993, Stepien et al. 1997) con nuevos ejemplares que presenten esta disminución en el número de parches de cirros nucales y cotejarlos con especímenes normales para establecer un posible origen de esta variación. Igualmente informativos pueden resultar estudios sobre conducta, bastante infrecuentes en peces de Chile.

\section{AGRADECIMIENTOS}

A Luis Hidalgo (Biblioteca, Museo Nacional de Historia Natural, Santiago) y a León Matamala M. (IZUA) por su apoyo a este estudio. Estos son resultados parciales del Proyecto S-200223 de la Dirección de Investigación de la Universidad Austral de Chile. 


\section{BIBLIOGRAFIA}

BATTS, B.S. 1964. Lepidology of the adult pleuronectiform fishes of Puget Sound, Washington. Copeia 1964 (4):666-673.

Castillo, R. \& G. Pequeño. 1998. Sinopsis de Triperygiidae de Chile (Osteichthyes: Perciformes). Gayana (Zool.) 62(2):109-133.

Cervigón, F., G. Pequeño \& C. Moreno. 1979. Descripción de Calliclinus nudiventris nov. sp. y notas adicionales sobre $C$. geniguttatus (Pisces: Clinidae) de Chile. Medio Ambiente. 4 (1):40-50.

HubBs, C. 1952. A contribution to the classification of the blennioid fishes of the Family Clinidae, with a partial revision of the Eastern Pacific forms. Stanford Ichthyol. Bull. 4 (2):41-158.

Hughes, D. 1981. Development and organization of the posterior field of ctenoid scales in the Platycephalidae. Copeia 1981 (3):596-606.

InzunZa, A. \& G Pequeño. 1988. Aspectos tróficoadaptativos y reafirmación de dos especies en el género Calliclinus Gill 1860 (Osteichthyes, Clinidae). Bol. Soc. Biol. Concepción 59:69-93.

Kotrschal, K. 1999. Sensory systems. In: Horn, M. H., K. L. Martin and M. A. Horn. M. H., K.L.M. Martin \& M.A Chotkowski (Eds.). 1999 Intertidal Fishes: Life in Two Worlds. Academic Press, San Diego, 399 pp.

Miles, M.C. \& J.I. Gaglione. 1992. A comparative study of Percid scales (Teleostei: Perciformes). Copeia 1992 (4):986-1001.
Pequeño, G. 1989. Peces de Chile. Lista sistemática revisada y comentada. Rev. Biol. Mar., Valparaíso 24:1-132.

RoberTs, C.D. 1993. Comparative morphology of spined scales and their phylogenetic significance in the Teleostei. Bull. Mar. Sci. 52 (1):60-113.

Smith-VAniz, W.F. \& V.G. Springer. 1971. Synopsis of the tribe Salariini with description of five new genera and three new species (Pisces: Blenniidae). Smith. Contr. Zool. 73: 1-72.

Stephens, J.S. \& V.G. SPRinger. 1974. Clinid fishes of Chile and Perú, with description of a new species, Myxodes ornatus, from Chile. Smith. Contr. Zool. 159:1-24.

Stepien, C. 1992. Evolution and biogeography of the Clinidae (Teleostei: Blennioidei). Copeia 1992 (2):375-392.

Stepien, C., M. Dixon \& D. Hillis. 1993 Evolutionary relationships of the Blennioid fish families Clinidae, Labrisomidae and Chaenopsidae: congruence between DNA sequence and allozyme data. Bull. Mar. Sci. 52 (1):496-515.

Stepien, C., A.K. Dillon, M.J. B rooks, K.L. Chase \&A.N. HuBERs. 1997. The evolution of blennioid fishes on an analysis of mitochondrial 12S r DNA. Mol. In: Kocher, T. D. \& C. A. Stepien (eds.). Academic Press, USA, Syst. Fish. 321 pp.

Von Bartheld, C.S. \& D.L. M EYer. 1985. Trigeminal and facial innervation of cirri in three teleost species. Cell Tissue Res. 241:615-622. 